\title{
Diacronie
}

Studi di Storia Contemporanea

$N^{\circ} 7,3 \mid 2011$

«Spagna Anno Zero»: la guerra come soluzione

\section{«Aviazione Legionaria»: il comando strategico- politico e tecnico-militare delle forze aeree italiane impiegate nel conflitto civile spagnolo}

\section{Edoardo Grassia}

\section{(2) OpenEdition \\ Journals}

Edizione digitale

URL: http://journals.openedition.org/diacronie/3411

DOI: 10.4000/diacronie.3411

ISSN: 2038-0925

Editore

Association culturelle Diacronie

Notizia bibliografica digitale

Edoardo Grassia, « «Aviazione Legionaria»: il comando strategico-politico e tecnico-militare delle forze aeree italiane impiegate nel conflitto civile spagnolo », Diacronie [Online], $N^{\circ} 7,3 \mid 2011$, documento 22 , Messo online il 29 juillet 2011, consultato il 30 avril 2019. URL : http://journals.openedition.org/ diacronie/3411 ; DOI : 10.4000/diacronie.3411 


\section{Diacronie}

N. 7 | 7|2011 Spagna Anno Zero: la guerra come soluzione

\section{2/}

\section{«Aviazione Legionaria»: il comando strategico-politico e tecnico-militare delle forzee aeree italiane impiegate nel conflitto civile spagnolo}

Edoardo GRASSIA*

Nel conflitto civile spagnolo intervennero 5699 militari e oltre 700 aerei della Regia Aeronautica italiana. Questa compagine, per non esporre bandiere o coccarde italiane vista la segretezza dell'intervento bellico in terra straniera, assunse il nome di Aviazione Legionaria. Nel mio articolo cercherò di mettere in luce le anomalie italiane del vertice politico-strategico che portarono alla decisione di intervenire nel conflitto spagnolo, visto che presero parte a questa decisione autorità estranee all'intervento militare e non intervennero, altresì, coloro che ne avevano piena autorità, nonché le forti stranezze circa l'impiego e il comando del "mezzo aereo", spesso utilizzato secondo il retaggio delle forze terrestri in sostituzione dell'artiglieria o dei carri armati. Considerando, infine, il conflitto civile spagnolo quale preludio della Seconda Guerra Mondiale, in conclusione porrò in luce come l'impegno assunto in Spagna apportò dei falsi insegnamenti dal punto di vista militare, per altro perpetuati nel più impegnativo conflitto mondiale, nonché la riconducibilità delle mancanze di materiale bellico lamentate dal duce italiano nel 1943 alla scarsa lungimiranza italiana nel suo impegno a fianco del Generalissimo Francisco Franco. 


\section{Premessa}

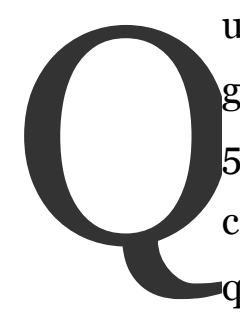

uando il regime fascista decise per l'aiuto militare alle forze nazionaliste guidate dal generale Francisco Franco, furono inviati nella penisola iberica 5699 militari della Regia Aeronautica', oltre a circa 700 aerei che, complessivamente, svolsero $134.889,46$ ore di volo ${ }^{2}$. Sul territorio spagnolo questa compagine assunse il nome di Aviazione Legionaria, cercando, in tal modo, di nascondere l'italica provenienza.

In relazione al processo che portò alla decisione di inviare in Spagna uomini e mezzi appartenenti alle regolari Forze Armate italiane possiamo notare la totale mancanza di fondamentali figure come il re o come i "tecnici" militari, custodi del reale stato dell'apparato bellico italiano. In termini di impiego del "mezzo aereo", invece, nonostante quanto già esso aveva dimostrato in precedenti impieghi di guerra, i velivoli operanti nei cieli spagnoli dovettero svolgere azioni proprie dell'artiglieria terrestre $o$, come fu scritto all'epoca, «in sostituzione dei cammelli dell'Intendenza» ${ }^{3}$.

\section{Introduzione}

Sin dagli inizi dell'Ottocento la Spagna fu teatro di una serie di guerre civili e colpi di Stato che opposero la città alla campagna, i costituzionalisti ai carlisti, il vecchio regime al nuovo, provocando una situazione nella quale il Governo mantenne con difficoltà il favore da parte dei propri cittadini ${ }^{4}$. In questo contesto l'esercito spagnolo divenne progressivamente un'istituzione semi-indipendente dal sistema politico ${ }^{5}$ e si fece più volte protagonista di molteplici atti di insubordinazione. In questi avvenimenti l'Italia non ebbe mai alcun ruolo ne si legò alla Spagna con trattati o alleanze.

I primi contatti tra Roma e Madrid vanno ricercati negli anni Venti del Novecento, quando furono firmati dei generici trattati di amicizia scaturiti principalmente dall'affinità ideologica del regime di Primo de Rivera con quello di Mussolini. Quando

\footnotetext{
${ }^{1}$ ROCHAT Giorgio, Le guerre italiane 1935-1943, Torino, Einaudi, 2005, p. 114.

${ }^{2}$ Stato Maggiore Aeronautica - Ufficio Storico - Operazione Militare Spagna (da adesso in poi SMA-US-OMS), Cartella 65.

${ }^{3}$ SMA-US-OMS, Cartella 96, Relazione del Generale Pricolo.

${ }_{5}^{4}$ BROWN, Harry, La guerra civile spagnola, Bologna, Il Mulino, 2000, pp. 7-8.

${ }^{5}$ Ibidem.
} 
però alle elezioni del gennaio 1936 il Fronte Popolare, che vide alleati repubblicani, anarchici, radicali, socialisti e comunisti, vinse le elezioni, i rapporti istituzionali tra i due Paesi si raffreddarono velocemente, con una parallela affermazione di quelli non istituzionali. In Spagna, la vittoria frentepopulista mise rapidamente in moto la macchinaria golpista che sollecitò aiuti economici e militari da quelle nazioni che, come l'Italia, potevano essere interessate alla creazione di un'alternativa politica avversa al Governo repubblicano.

Non entrando nel merito delle 'cause' di quanto accadde tra il 17 e il 18 luglio 1936, prima nel Marocco spagnolo e poi nella penisola iberica, sappiamo oggi che anche in precedenza i militari golpisti spagnoli avevano cercato aiuto in Italia. Nell'aprile 1932, in occasione del pronunciamiento del Generale Sanjurio, il Maggiore dell'Aeronautica spagnola Juan Antonio Ansaldo fu inviato a chiedere aiuto a Italo Balbo ${ }^{6}$; nell'autunno 1933 ancora Ansaldo insieme a Calvo Sotelo incontrò nuovamente il maresciallo Balbo per concordare aiuti in caso di insurrezione antirepubblicana ${ }^{7}$; il 31 marzo 1934 Antonio Goicoechea, anziano leader del gruppo monarchico, Antonio Lirarza Irribarren, leader navarrese dei carlisti, il nazionalista Rafael de Olazaben ed il Generale Emilio Barrera, ex Capo di Stato Maggiore dell'Esercito spagnolo, si recarono da Mussolini per richiedere fondi, armi e l'addestramento di truppe spagnole in Italia ${ }^{8}$.

Per l'Alzamiento del luglio 1936, la prima richiesta di aiuto avanzata dai golpisti all'Italia fu visionata da Mussolini il 20 luglio, ma il duce italiano rispose negativamente ${ }^{9}$. Fallito il tentativo con il massimo dirigente, il giorno seguente, si tentò con il Ministro degli Affari Esteri Galeazzo Ciano, ma anche questa volta ci fu un rifiuto. Nonostante l'apparente disinteresse iniziale, le trattative seguirono fino a capovolgere la posizione sino ad allora sostenuta; a ciò contribuirono anche le informazioni che giungevano circa possibili aiuti francesi al fronte repubblicano, motivo per cui Mussolini, spronato dal Ministro Ciano, decise per l'aiuto militare del fronte nazionalista ${ }^{10}$.

Sotto mentite spoglie, senza insegne italiche e con una falsa transazione economica di acquisto dalla nota ditta SIAI - Marchetti, il giorno 30 luglio dodici bombardieri

\footnotetext{
${ }^{6}$ RANZATO, Gabriele, L'eclissi della democrazia. La guerra civile spagnola e le sue origini 1931-1939, Torino, Bollati Boringhieri, 2004, nota 13, p. 8.

${ }^{7}$ COVERDALE, John F., I fascisti italiani nella guerra civile di Spagna, Roma-Bari, Laterza, 1977, p. 44.

${ }^{8}$ LIZARZA, Antonio, Memories de las cospiracion, Pamplona, 1953 in THOMAS, Hugh , Storia della guerra civile spagnola, Torino, Giulio Einaudi Editore, 1963, p. 89, e COVERDALE, John F., op. cit., p. 46. THOMAS, citando LIZARZA, ci parla di 400 giovani carlisti addestrati in Italia, mentre COVERDALE ne cita tre gruppi di circa quindici persone ciascuno.

${ }^{9}$ COVERDALE, John F., op. cit., p.17.

${ }^{10}$ THOMAS, Hugh, op. cit., nota 3, p. 239.
} 
SM.81 regolari della Regia Aeronautica, partirono dalla base di Elmas, in Sardegna, alla volta del Marocco spagnolo per il trasporto dell'Esercito d'Africa sul continente europeo. Dei dodici, solo nove giunsero a destinazione: il bilancio della prima missione italiana in Spagna si sarebbe conclusa con un totale di tre velivoli persi e quindici morti. Queste prime statistiche ci rendono conto delle difficoltà iniziali della campagna militare che l'Italia intraprese in terra iberica ${ }^{11}$.

L’intervento italiano in territorio straniero - nonostante la segretezza e l'iniziale breve durata della missione - assunse dimensioni sempre maggiori. Relativamente a quanto portò alla decisione di appoggiare le forze nazionaliste, non possiamo ipotizzare manifestazioni di interventismo o neutralismo e, ancor meno, a dibattiti politici e parlamentari, vista la natura del regime al potere in Italia. Sarebbe lecito ipotizzare un nucleo decisionale con il re, Supremo Capo delle Forze Armate, il Presidente del Consiglio, i vari Ministeri come Difesa, Economia e Industria ed Esteri, e, soprattutto, le più alte cariche militari, come il Capo di Stato Maggiore Generale, i Capi di Stato Maggiore delle singole Forze Armate e dell'intelligence. Questi ultimi avrebbero potuto dare indicazioni circa il reale stato delle Forze Armate italiane e degli apparati bellici delle altre nazioni europee quando, visto il clima di crescente militarizzazione delle potenze del vecchio continente ${ }^{12}$, era già ipotizzabile un nuovo conflitto di vaste dimensioni.

Con la consapevolezza che le dittature non amano l'ordinaria amministrazione ${ }^{13}$ sorgono oggi una serie di interrogativi relativamente a come venne deciso l'intervento militare in Spagna e com fu strutturata la gerarchia di comando, soprattutto alla luce del fatto che, intendendo il conflitto civile spagnolo come preludio della Seconda Guerra Mondiale, le decisioni e le procedure attuate in Spagna furono erroneamente interpretate come dei reali successi politici e militari e, in quanto tali, fortemente pubblicizzati dal regime e perpetuati nella loro applicazione proprio nel successivo conflitto mondiale.

\section{L'intervento e la linea di comando politico-strategico}

\footnotetext{
${ }^{11}$ GRASSIA, Edoardo, L'Aviazione Legionaria da bombardamento. Spagna 1936-1939. Iniziare da stanotte azione violenta su Barcellona, Roma, IBN Editore, 2009, p. 96.

${ }^{12}$ KENNEDY, Paul, Ascesa e declino delle grandi potenze, Milano, Garzanti Editore, 1989, nota p. 161, p.778, citando WRIGHT, Quincy, Study of war, Midway Reprint, p. 672, propone delle statistiche comparative tra Francia, Germania, Gran Bretagna, Russa ed Italia in ambito economico e militare nel periodo anni Trenta. Da esse è facile rilevare come l'Italia fosse la nazione meno preparata economicamente e militarmente ad affrontare un conflitto di grandi dimensioni. Anche in GRASSIA, Edoardo, op. cit., pp. 34-44.

${ }^{13}$ MONTANELLI, Indro, CERVI, Mario, Storia d'Italia vol. XLII. La guerra di Spagna e il patto di Monaco, Milano, Rizzoli, 1984, p. 7.
} 
Gli artefici dell'intervento bellico furono esclusivamente il duce e il suo Ministro degli Esteri, Galeazzo Ciano. Proprio quest'ultimo, il 28 agosto 1937, in relazione all'appoggio italiano alle forze nazionaliste, scrisse nel segreto del suo diario: «Questa impresa di Spagna trova la costante opposizione della Marina, che fa resistenza passiva. L'Aeronautica, benissimo. L'Esercito con regolarità. La Milizia con slancio. Ma in fondo il duce ed io soli ne siamo i responsabili: anzi, coloro che ne hanno il merito. Un giorno si riconoscerà che è grande» ${ }^{14}$. Oggi possiamo considerare totalmente sbagliata la predizione fatta da Ciano in termini di 'merito'. La scelta iniziale di assumere tale impegno militare derivò, probabilmente, da una serie di errate considerazioni. Il conflitto si pensò di breve durata e, dal punto di vista militare, poteva essere un'occasione da sfruttare come luogo per l'addestramento degli uomini nonché come un momento interessante per la sperimentazione di tecniche e materiali. Si pensò, come scrisse Dino Grandi nel suo diario ${ }^{15}$, che una vittoria repubblicana avrebbe aperto il dominio francese nel Mediterraneo che, invece, nell'ottica del mare nostrum, costituiva uno dei principali obbiettivi di Mussolini ${ }^{16}$. Probabilmente si pensò alla possibilità di convertire la Spagna di Franco in un futuro alleato considerata l'avversione di Mussolini per le repubbliche ${ }^{17}$, ma anche ad un veloce accrescimento del peso internazionale dell'Italia.

$\mathrm{Si}$ può considerare che la partecipazione militare internazionale, per la sua fattibilità, per la sua efficacia e per la sua organizzazione complessiva, avrebbe dovuto richiedere una più ampia riflessione coinvolgendo un maggior numero di autorità politiche e militari. Appare il caso sottolineare - come già detto - che, dal punto di vista militare, le scorte di materiale bellico erano già basse visto il quasi concomitante

\footnotetext{
${ }^{14}$ CIANO, Galeazzo, Diario 1937-1943, Milano, Rizzoli Editore, 1980, giorno 28 agosto 1937, p. 29.

${ }^{15}$ Riportato nell'introduzione di DE FELICE, Renzo, al testo di COVERDALE, John F., I fascisti italiani nella guerra di Spagna, op. cit., p. XVII

${ }_{16}$ I $^{\mathrm{O}}$ Convegno Nazionale per gli Studi di Politica Estera, Gli interessi dell'Italia nel mediterraneo orientale, (Milano, 15-17 ottobre 1936) relatore PACE, Biagio: «[...] la presenza di interessi italiani (nel Mediterraneo ndr) dura ininterrotta da quando una unità civile è apparsa nel nostro mare». Per quanto il Convegno sia afferente al Mediterraneo orientale, più volte il relatore parla di Mediterraneo in termini generali, coinvolgendo anche collocazioni che sono ad occidente nel definire gli interessi territoriali, economici, marittimi, ecc., del regime fascista. Istituto per gli Studi di Politica Internazionale. Disponibile al sito URL: < http://cronologia.leonardo.it/storia/a1936xk.jpg > [consultato il 03.05.2007].

${ }^{17}$ Proprio nell'occasione della vittoria del Fronte Popolare in Spagna Mussolini disse che «fare una repubblica oggi è come usare la lampada a petrolio al tempo della luce elettrica». Riportato in MONTANELLI, Indro, CERVI, Mario, Storia d'Italia vol. XLII. La guerra di Spagna e il patto di Monaco, op. cit., p. 9.
} 
impegno militare italiano nella guerra d'Etiopia ${ }^{18}$; inoltre, visto il panorama internazionale, non si poteva dimenticare la possibilità di un nuovo conflitto di più vaste dimensioni.

Analizzando a grandi linee il vertice politico-strategico che avrebbe dovuto intervenire in una decisione così importante, facendo un particolare riferimento alle forze aeree, possiamo rilevare che:

- il Re; essendo il Capo Supremo dello Stato e avendo il comando delle Forze Armate avrebbe dovuto essere il primo attore nel consentire che l'Italia prendesse parte ad un nuovo impegno bellico. Come noto, Mussolini tese sempre ad oscurare la figura del monarca con una serie di prerogative e, tra di esse, l'impiego delle Forze Armate. Vittorio Emanuele III fu tagliato fuori da ogni decisione relativa alle azioni di guerra italiane degli anni Trenta. Se l'intervento militare in Etiopia fu perpetrato nonostante il monarca fosse di parere contrario, per quanto attiene a quello spagnolo non venne per nulla interpellato, ragione per cui si possa escludere il re dalla linea di comando;

- Mussolini; contemporaneamente Capo del Governo, Ministro della Guerra e Ministro dell'Aeronautica. Per ovvi motivi il primo incarico lo proiettò al vertice politico-strategico, mentre le altre due cariche ricoperte gli diedero diritto di entrare nella gerarchia militare della missione in terra iberica e nella linea di comando dell'Aviazione Legionaria. Nonostante ciò, il duce intervenne spesso in maniera “un po' troppo diretta”, saltando spesso i canali ed i livelli di comando. Ciò va letto in funzione del fatto che, nelle Forze Armate come in altre amministrazioni, il duce volle sempre un contatto diretto con le basi in modo tale da non creare potenze personali intermedie tra sè e le forze operative che potessero in qualche modo offuscare la sua autorità. Lo dobbiamo considerare come l'apice della struttura gerarchica della Missione Militare Italiana in Spagna (da ora in poi MMIS, così come nei documenti ufficiali) e, di conseguenza, delle forze aeree in essa impiegate;

- Galeazzo Ciano; Ministro degli Affari Esteri all'età di trentatrè anni. Per il suo incarico avrebbe dovuto dirigere solo la diplomazia italiana, ma non fu così. Occorre considerarlo fulcro dell'impegno militare italiano in Spagna non solo in relazione alla decisione di intervenire militarmente di cui, come visto, si autocelebrò anche nel suo

\footnotetext{
${ }^{18}$ Tra la campagna d'Africa e la missione in Spagna la Regia Aeronautica impiegò oltre 1600 aerei di vario tipo che, chiaramente, vennero a mancare nel Secondo Conflitto Mondiale. Nella sola guerra civile spagnola, inoltre, la Regia Aeronautica ebbe un onere finanziario di 1.754.739.658,24 di lire come desunto da SMA-US-OMS- Cartella 97 - "Relazione contabile riguardante la fornitura di materiali per l'esigenza O.M.S. da parte dell'Amministrazione Aeronautica”.
} 
diario $^{19}$, ma anche in relazione alla gestione delle forze combattenti. Per volontà del duce, inoltre, presso il Ministero degli Esteri (d'ora in poi MAE) fu istituito l'Ufficio Spagna, cuore pulsante dell'intervento militare nella penisola iberica. Ciano, quindi, che avrebbe dovuto essere personaggio esterno alla linea di comando militare, deve essere considerato al secondo posto della gerarchia. Dal suo diario possiamo apprendere come intervenne, spesso personalmente, nell'emanazione di ordini tanto alle forze di terra quanto ai reparti di volo, nonché nell'attribuzione di particolari incarichi di comando. Leggendo le sue memorie, possiamo trovare affermazioni quali: «Ho dato ordine di bombardare stanotte Valencia con gli aerei di Palma. Bisogna cogliere il momento per terrorizzare il nemico ${ }^{20} ;$ «Ho visto Cupini [aviatore] reduce dalla vittoria aerea di Damasco. Mi ha chiesto un comando in Spagna e l'ho subito accontentato ${ }^{21}$; «il Generale Gambara [comandante di tutte le forze terrestri ed aeree italiane in Spagna] ritiene possibile far massa e sfondare nella direzione di Valenza. Gli ho detto di agire senz'altro ${ }^{22}$;

- il Generale Pietro Badoglio; dopo la vittoria in Etiopia, fu nominato Capo di Stato Maggiore Generale e avrebbe dovuto essere il punto di comando, coordinamento e controllo delle Forze Armate impiegate in Spagna o operanti in segreto nelle acque del Mediterraneo. In realtà egli non andò mai oltre un puro ruolo di rappresentanza pagando, probabilmente, la sua nota fedeltà alla monarchia. Mai interpellato per decidere sull'intervento militare, ricevette informazioni dallo Stato Maggiore del Regio Esercito a titolo di cortesia. Avrebbe dovuto essere il vertice del comando militare e il primo responsabile degli ordini delle operazioni belliche, della condotta delle forze impiegate e delle nomine degli uomini di comando, ma fu invece escluso.

- Il Generale Giuseppe Valle; dopo l'allontanamento del maresciallo Balbo in Libia, assunse la carica di Sottosegretario alla Regia Aeronautica e Capo di Stato Maggiore della stessa. Fu un personaggio un po' altalenante per quanto concerne le operazioni aeree in Spagna. Inizialmente, con un gesto totalmente sconsiderato, accompagnò i primi dodici aerei alla volta del Marocco spagnolo, senza pensare alle conseguenze che si sarebbero potute verificare se, per qualsiasi motivo, dall'avaria all'incidente, fosse dovuto atterrare in territorio francese. Come spiegare la presenza di un personaggio di vertice delle Forze Armate italiane con aerei riverniciati e con equipaggi dotati di falsi documenti? Ma oltre a questa prestazione, relativamente al

\footnotetext{
${ }^{19}$ CIANO, Galeazzo, op. cit., p. 29.

${ }^{20}$ Ibidem.

${ }^{21}$ Ibidem.

${ }^{22}$ Ibidem, p. 147.
} 
conflitto spagnolo e per quanto concerne l'attività decisionale, Valle ricadde nell'ombra. Solo nelle occasioni in cui Mussolini ordinò qualcosa lui provvide alla diramazione degli ordini; quando giunsero dai comandi delle forze aeree lamentele o indicazioni di gravi problematiche, spesso legate alla dipendenza e all'impiego disposti da comandanti di truppe terrestri, non provvide a svolgere alcuna pressione sul duce per sostenere le cause del suo personale. Come illustrato in precedenza, Cupini per ottenere un comando aeronautico nella missione spagnola si rivolse a Ciano, del dicastero degli esteri, e non a lui che era il vertice della Forza Armata aerea.

Riassumendo, il fulcro politico-strategico che assunse la decisione di intervenire militarmente nel conflitto civile spagnolo fu rappresentato da Mussolini e Ciano, con attività di coordinamento e di informazione svolte dall'Ufficio Spagna presso il MAE. Tutti questi, oltre alla veste politica dell'impresa spagnola, assunsero anche un ruolo non secondario nel diretto impiego delle forze aeree inviate procedendo all'emanazione di ordini diretti ai reparti dislocati nella penisola iberica e nelle isole Baleari.

\section{La linea di comando nel teatro di guerra}

La delicatezza della situazione nel teatro di guerra spagnolo risentì chiaramente delle problematiche connesse al comando di vertice politico-strategico sopra discusse. Ma per la gestione del coordinamento, del comando e del controllo delle forze italiane impiegate nella penisola iberica vi furono problemi ancor maggiori dovuti alla scarsa standardizzazione e alla non precisa definizione di compiti e responsabilità dovuti, soprattutto, al sistema di attribuzione degli incarichi di comando. 


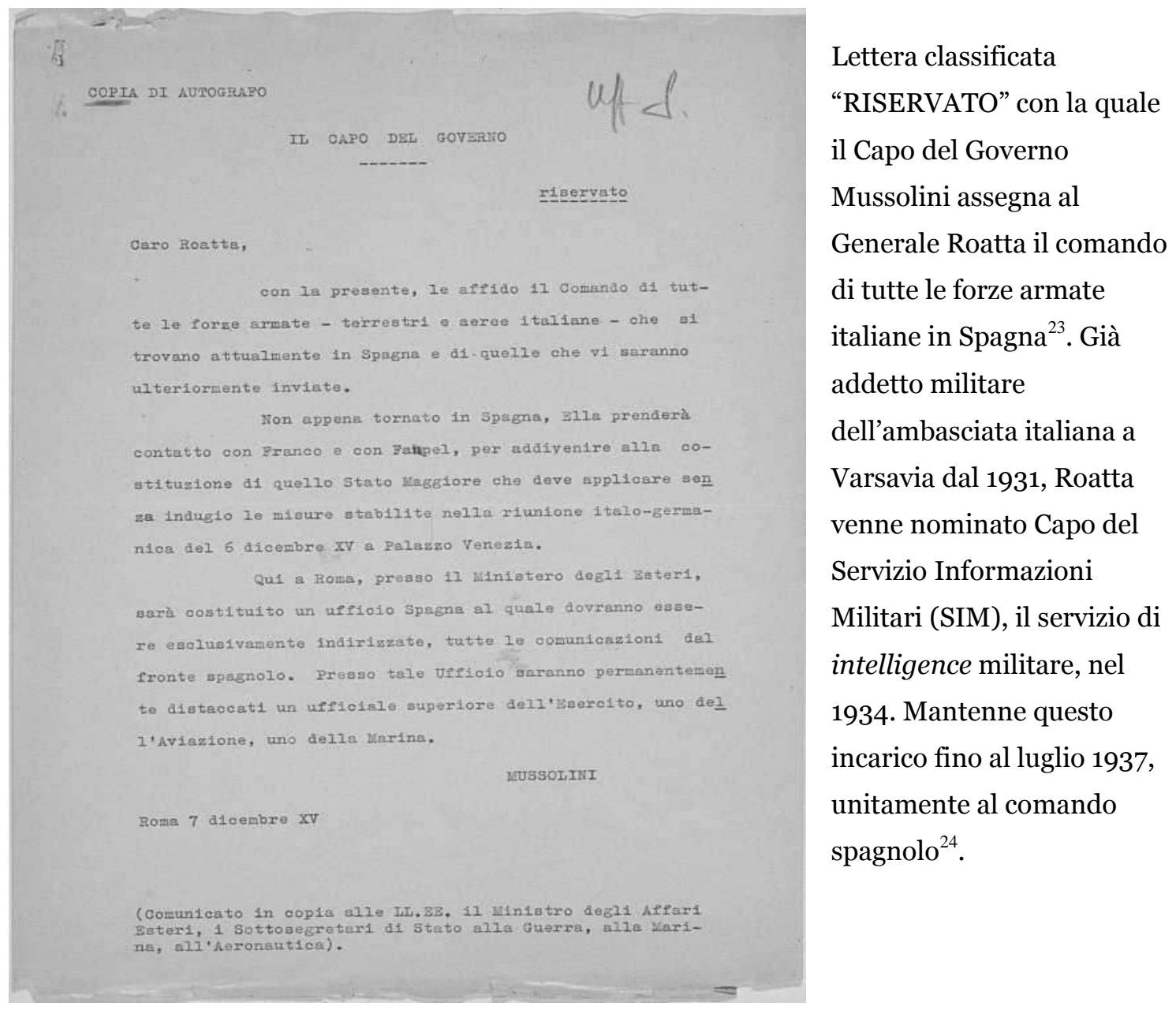

Senza alcuna proposta delle autorità militari, Mussolini dispose un incarico di alto comando con una semplice lettera che, in copia, venne inviata al Ministro degli Affari Esteri e ai Sottosegretari di Stato alla Guerra, alla Marina e all'Aviazione. Non ci fu invece nessuna comunicazione per il re o per il Capo di Stato Maggiore Generale Badoglio.

Impiegati nella MMIS possiamo trovare reparti regolari del Regio Esercito, forze della Milizia Volontaria arruolata attraverso i canali del Partito Nazionale Fascista e reparti di volo da caccia, da bombardamento e da ricognizione della Regia Aeronautica, tutti sotto mentite spoglie, privati di stellette e con falsi documenti. I vertici di tutte queste componenti erano Ufficiali del Regio Esercito, Ufficiali della Milizia ovvero gerarchi fascisti che in teatro di guerra vestirono i gradi da generale e ufficiali della Regia Aeronautica. Tutti, il più delle volte, riuscirono ad ottenere un incarico nell'ambita missione spagnola grazie a un contatto diretto con il Capo del Governo o

${ }^{23}$ SMA-US-OMS - Cartella 73. Lettera "RISERVATO”, del 7 dicembre 1936, firmata dal Capo del Governo Mussolini.

${ }^{24}$ MUNZI, Ulderico, Il Generale, Costabissara, Angelo Calla Editore, 2009, p. 295. 
con il Ministro degli Affari Esteri, ma non necessariamente con i vertici delle strutture militari. Per quanto riguarda la nomine del Comandante dell'intera MMIS, la logica derivante da precedenti esperienze belliche $\mathrm{e}^{25}$ avrebbe fatto presupporre la proposta di un qualificato ufficiale da parte del Capo di Stato Maggiore Generale Badoglio o dei Capi di Stato Maggiore delle singole Forze Armate. Già abbiamo discusso, in realtà, come questi furono tagliati fuori da qualsiasi decisione circa il conflitto civile spagnolo.

In realtà, sempre perché non si volle seguire l'ordinaria amministrazione e nella volontà di avere un rapporto diretto con la base, privo di organi e uomini intermedi che avrebbero potuto, ex post, acquisire notorietà e potere, Mussolini decise di conferire il comando di tutti gli italiani in Spagna con una lettera. "Caro Roatta», è questo l'inizio della missiva che Mussolini scrisse il 7 dicembre 1936. Con grande familiarità ed in totale autonomia dai vertici militari, il duce scrisse una "semplice" lettera per indicare il comando delle forze italiane in Spagna scegliendo, per tale incarico, un Generale di Brigata del Regio Esercito. Appena promosso al grado di Generale, questi comandò quattro divisioni terrestri e tutte le forze aeree composte da quattro Stormi, quattro Gruppi Autonomi di volo, una Sezione idrovolanti e una Squadriglia trasporti. La scelta diretta cadde su di un uomo del regime che, anche dopo il conflitto mondiale, si legò alla Spagna franchista ${ }^{26}$. «Caro Roatta», dunque, «con la presente le affido il Comando di tutte le forze armate - terrestri ed aeree - che si trovano attualmente in Spagna e di quelle che vi saranno ulteriormente inviate». ${ }^{27}$

Un Generale di Brigata dell'Esercito, portatore del retaggio, della preparazione e della cultura propria dei comandanti delle forze di terra venne, quindi, posto al comando anche dell'Aviazione Legionaria. Questa situazione, in particolare, va letta con i caratteri propri di quel periodo dove la Regia Aeronautica, autonoma da appena un decennio, non era ancora ben accetta dalle altre due Forze Armate: Regio Esercito e Regia Marina. Nonostante le dimostrazioni belliche del primo conflitto mondiale e della guerra d'Etiopia $^{28}$, e i conseguenti innumerevoli studi di tecnica di guerra aerea ${ }^{29}$,

${ }^{25}$ PELLICCIA, Antonio, La regia Aeronautica, dalle origini alla seconda guerra mondiale (1923-1943), Roma, Stato Maggiore dell'Aeronautica - Ufficio Storico, 1992, p. 33. Balbo, in uno studio scritto nel 1933 sulla riorganizzazione delle Forze Armate, previde un Capo di Stato Maggiore unico con piena autorità su Esercito, Marina e Aeronautica. Lo studio cadde inascoltato.

${ }^{26}$ Il 16 novembre 1944 il Generale Roatta fu arrestato per l'inchiesta sulla mancata difesa di Roma e, l'anno successivo, fu indagato anche per l'assassinio dei fratelli Rosselli. Il 4 marzo 1945 Roatta evase dal carcere militare realizzato presso l'istituto Virgilio a Roma e, con la moglie, si mise definitivamente in salvo proprio in Spagna grazie al Generale Francisco Franco.

${ }^{27}$ SMA-US-OMS - Cartella 73. Lettera "RISERVATO", del 7 dicembre 1936, firmata dal Capo del Governo Mussolini.

${ }^{28}$ Il Generale Pietro Badoglio, allora Capo di Stato Maggiore Generale, scrisse in "La Guerra d'Etiopia", del 1936: «l'Aviazione era stata presente in tutte le fasi della guerra e in ogni fase di 
l'aviazione era ancora considerata come una punta di diamante e non un elemento risolutivo dei conflitti, ovvero, una concorrente nella divisione del bilancio destinato al comparto di difesa. Roatta, probabilmente, sapeva di non poter avere un grande ascendente sull'Aviazione Legionaria e, sin dal principio, questa dipendenza non fu priva di tensioni. Per lui la possibilità di riuscire nel delicato comando fu perciò percepita attraverso il filtro che poteva creare il nome del duce. Il Generale, infatti, pensò bene di attrincerarsi dietro la massima autorità convinto che nessuno avrebbe contestato gli ordini e le indicazioni di Mussolini. Nella lettera del 16 dicembre 1936, prot. 847, classificata «SEGRETO» indirizzata al Comandante Gallo, alias Colonnello Bonomi, primo comandante dell'Aviazione Legionaria, Roatta scrisse:

Avendo io, per ordine di S.E. il Capo del Govemo, assunto il Comando di tatte le forze armate - terrestri ed aeree italiane -

Particolare della lettera 847 del 16.12.1936 con la specificazione della volontà di S.E. il Capo del Governo $^{30}$.

«Avendo io, per ordine di S.E. il Capo del Governo, assunto il Comando di tutte le forze armate - terrestri ed aeree italiane $-[\ldots] » .{ }^{31}$ Mettendosi sotto il tranquillo riparo di Sua Eccellenza il Capo del Governo, Mario Roatta assunse anche il comando dell'Aviazione Legionaria e, quindi, ne indicò i compiti con due lettere.

L'interesse per il primo scritto, lettera prot. n. 895 del 18 dicembre $1936^{32}$, nasce dal fatto che con essa si realizzò, innanzitutto, una separazione ufficiale tra l'Aviazione Legionaria Spagna e l'Aviazione Legionaria delle Baleari. Nell'indicare i compiti dell'Aviazione dislocata nella Spagna continentale, il Comandante del CTV, ne parlò in termini di «aviazione d'esercito», palesando in tal modo quale fosse il suo pensiero di dottrina di impiego del mezzo aereo ancora classificato e impiegato, come in passato, in qualità di componente del Regio Esercito, snaturando e impiegando malamente le potenzialità belliche dei velivoli ormai riconosciute in tutto il mondo e creando, da subito, astio con i vertici dei reparti di volo.

ogni singola battaglia [...] è l'arma dell'avvenire [...] ma tanto più renderà quanto più strettamente agirà in coordinamento con l'Esercito» citato in PELLICCIA Antonio, op. cit., p. 71.

${ }^{29} \mathrm{Si}$ pensi, a tal proposito, al fatto che la dottrina di guerra aerea di Giulio Douhet aveva ormai fama mondiale.

${ }^{30}$ SMA-US-OMS- Cartella 46, lettera 847 del 16.12.1936.

${ }^{31}$ Ibidem.

${ }^{32}$ SMA-US-OMS- Cartella 46, lettera 895 del 18.12.1936. 


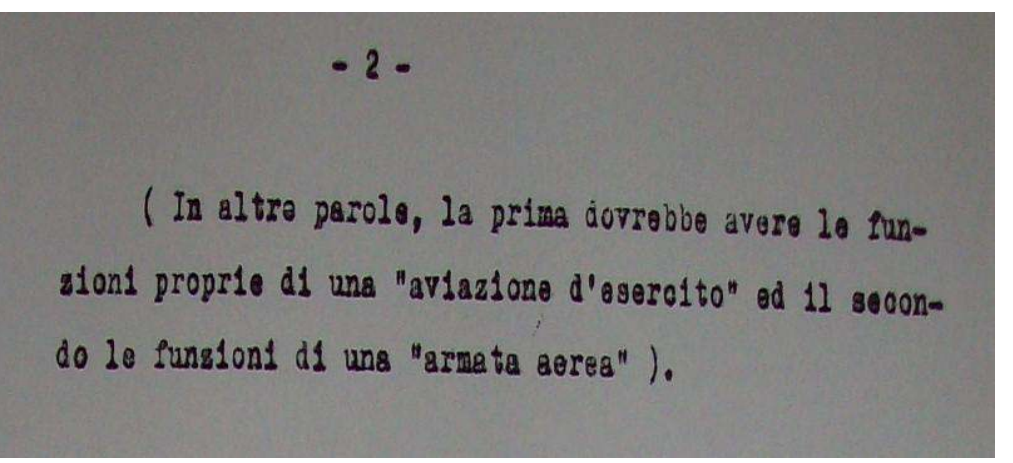

Particolare della lettera 895 del 18.12.1936 dove si specificano le funzioni dell'Aviazione Legionaria: aviazione d'esercito ${ }^{33}$.

Tra le principali conseguenze, per altro spesso rilevate in relazioni scritte dai vertici dell'Aviazione Legionaria per il Sottosegretario Generale Valle, ma rimaste inascoltate, occorre sottolineare l'impiego fatto dei reparti di bombardamento: "in sostituzione dell'artiglieria”. Comandati a svolgere azioni belliche a bassa quota, azioni per le quali né gli aerei erano predisposti, né i piloti addestrati, si trovarono spesso a doversi confrontare non solo con la contraerea repubblicana, ma con le più semplici armi individuali.

Per quanto concerne l'Aviazione Legionaria delle Baleari, invece, Roatta scrisse semplicemente che essa svolgerà delle azioni «in base a disposizioni di questa Missione» ${ }^{34}$. Un ordine estremamente generico, quasi a non sapere quali impieghi tattici o strategici affidare alla componente aerea. In realtà, per quanto attiene all'Aviazione Legionaria delle Baleari, gli ordini di missione giunsero molto spesso direttamente da Mussolini e da Ciano, come è facile rilevare proprio nelle pagine del diario scritto da quest'ultimo. Si trattò tanto di ordini di bombardamento, come quelli che devastarono la città di Barcellona nel marzo 1938, quanto di ordini di sospensione dei bombardamenti, per esortare Francisco Franco a svolgere azioni risolutive per il conflitto.35 Anche in questo caso occorre rilevare l'anomalia di questa linea di comando. A Roma, anche se presso l'Ufficio Spagna giungevano i rapporti della guerra spagnola, non si poteva avere una piena conoscenza delle condizioni esistenti, considerata sia l'efficacia dei mezzi di comunicazione sia la realtà e la completezza dei rapporti stessi. Si rischiava di dare degli imperativi estremamente generici che aumentavano le già difficili condizioni di impiego dei mezzi e del personale. E' il caso, per esempio, dell'ordine di effettuare un «martellamento costante e diluito nel tempo sulla città di

\footnotetext{
${ }^{33}$ Ibidem.

${ }^{34}$ SMA-US-OMS- Cartella 46, lettera 895 del 18.12.1936.

${ }^{35}$ CIANO, Galeazzo, op. cit. Nel testo si incontrano molto spesso delle indicazioni che Ciano o Mussolini danno ai comandanti territoriali in Spagna sulla condotta della guerra. Per l'Aviazione Legionaria si vedano in particolare pp. 92, 95, 96, 104 e 115.
} 
Barcellona», comandato da Mussolini al Generale Valle il 16 marzo 193836. Di fronte a tale dispaccio si poteva colpire la città o l'importante porto, la stazione che era un nodo fondamentale di smistamento delle truppe o i quartieri abitati dai civili, si poteva cercare di fiaccare il morale o spezzarne l'economia. "Diluito nel tempo", inoltre, poteva significare un lasso totalmente soggettivo. Appaiono evidenti le difficoltà di chiunque dovesse materialmente mettere in pratica tale direttiva.

Tornando all'azione di comando del Generale Roatta, possiamo dire che esso ebbe concretamente il comando di metà dell'Aviazione Legionaria, quella continentale, mentre le principali direttive dell'altra metà, quella isolana delle Baleari, fu esercitato direttamente da Roma. Entrambe queste metà, come visto, ebbero differenti problemi di impiego che, logicamente, si andarono a ripercuotere in maniera più o meno incisiva sulle potenzialità belliche, sui rapporti con le altre forze alleate tedesche e nazionaliste, nonché sulle condizioni di pericolo in cui i piloti dovettero affrontare le proprie azioni per eseguire gli ordini ricevuti.

${ }^{36}$ CIANO, Galeazzo, op. cit., p. 115, e SMA-US-OMS - Cartella 75. Telegramma del 16 marzo 1938. 
Relativamente

al secondo scritto, lettera prot. 922 non datata ${ }^{38}$, occorre rilevare come a distanza di poche righe si ribadisca prima che «Il Comando dell' Aviazione Legionaria è insediato presso la MMIS»; ovvero, che l'Aviazione Legionaria veniva organicamente considerata come un qualsiasi altro reparto del Regio Esercito o della Milizia Volontaria senza alcuna considerazione del suo status di Forza Armata autonoma e, poco dopo, che «Il Comandante dell'A.L., che è alle dipendenze del Capo della M.M.I.S [...]»; dove, evidentemente, si sentiva la necessità di ribadire un vincolo di dipendenza

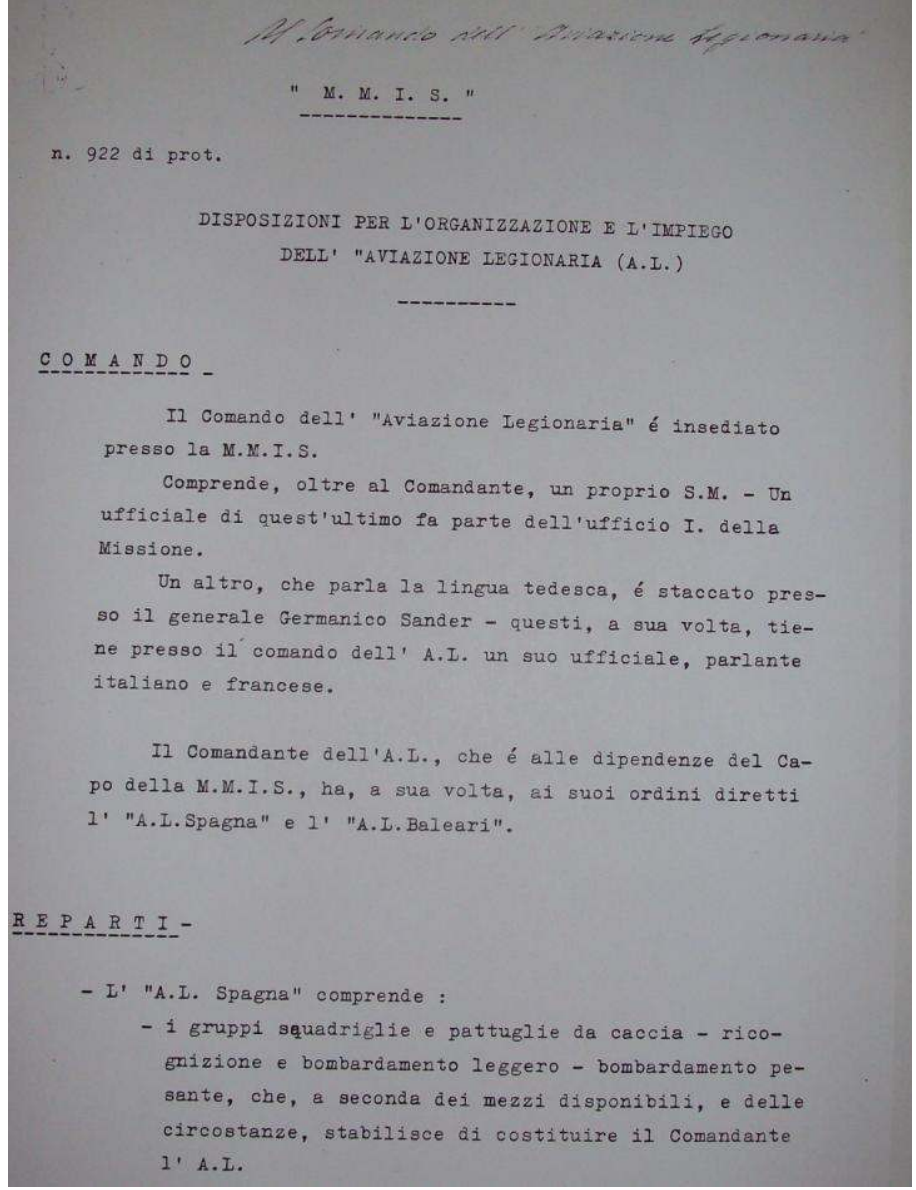

Lettera prot 922, con la quale il Generale di Brigata Mario Roatta, Comandante della MMIS, comunica le sue disposizioni tanto per l'organizzazione quanto per l'impiego dell'Aviazione Legionaria ${ }^{37}$. gerarchica comunque già nota. Continuando l'analisi del vertice militare della MMIS, la linea di comando si fece particolarmente problematica quando il Generale di Divisione Aerea Mario Bernasconi assunse il comando dell'Aviazione Legionaria. Ci si trovò di fronte ad una situazione dove Roatta, Generale di Brigata, aveva il comando su di un Generale di Divisione, ovvero militarmente superiore. Una situazione insostenibile sanata solo quando anche il primo fu sostituito dal Generale di Corpo d'Armata Ettore Bastico, a sua volta superiore di Bernasconi.

Oltre all'annullamento della fondamentale gerarchia militare verificatosi con la contemporanea presenza del binomio Roatta-Bernasconi, la dipendenza gerarchica da un comandante terrestre fu più volte oggetto di lamentele da parte dei piloti italiani.

\footnotetext{
${ }^{37}$ SMA-US-OMS- Cartella 46, lettera 922, senza data.

${ }^{38}$ Ibidem.
} 
Tra queste consideriamo lo scritto che il Generale Vincenzo Velardi, il 6 maggio 1937, indirizzò al Sottosegretario dell'Aeronautica e Capo di Stato Maggiore della stessa forza armata Generale Valle ${ }^{39}$. Velardi lamentò il fatto che il Comandante del CTV, non solo rendeva i rapporti con l'Aviazione Legionaria sempre più difficoltosi ma creava problemi anche per il funzionamento dei reparti da osservazione aerea e per l'impiego di quelli da caccia e da bombardamento ${ }^{40}$. «Cerca ogni minima occasione per far pesare comunque la propria arbitraria invadenza e per tentare di soffocare l'azione di comando dello scrivente», proseguiva il Generale Velardi riferendosi a Mario Roatta con un vero e proprio atto di accusa che faceva ipotizzare il raggiungimento di una situazione non più sostenibile e che, con tutta probabilità, si sarebbe protratta e forse accentuata nel futuro. Una situazione, ancora, che non permetteva l'adesione dell'Aviazione Legionaria alle azioni aeree di concorso con le forze aeree tedesche e spagnole, o all'effettuazione di azioni di massa o di azioni «politicamente importanti».

Velardi, ancora, lamentò il fatto che il sottostare all'azione di comando svolta dal Comandante del CTV difficoltò l'opera di avvicinamento, coordinamento e collaborazione delle «tre aviazioni amiche», italiana, tedesca e spagnola. Persino il contatto diretto tra il Comandante dell'Aviazione Legionaria e il Sottosegretario per la Regia Aeronautica era stato formalmente proibito. Il Generale Velardi, nel suo scritto, dichiarò espressamente di «non ritenere che l'Aviazione Legionaria dovesse dipendere da un Generale dell'Esercito» richiedendo perciò l'indipendenza della sua azione di comando. Il promemoria per il Sottosegretario di Stato Generale Valle, essendo un documento indirizzato ad personam, sarebbe dovuto restare tale. All'alta autorità si richiedeva una ben precisa azione in ambito di vertice politico-strategico. Ma la sorpresa arrivò il successivo 30 aprile 1937. La comunicazione del Generale Velardi, giunse fortuitamente anche al MAE che, a sua volta, la girò al Comandante del CTV.

La reazione di quest'ultimo fu molto forte. Con Messaggio cifrato 5788 del 30 aprile $1937^{41}$, Roatta scrisse a Velardi: « [...] darmi comunicazione integrale di quanto trasmesso et ragioni per le quali S.V. che est completamente mia dipendenza avrebbe saltato mio tramite».

\footnotetext{
${ }^{39}$ SMA-US-OMS - Cartella 73 - Promemoria per il Sottosegretario di Stato.

${ }^{40}$ GRASSIA, Edoardo, op. cit., pp. 73-77.

${ }^{41}$ SMA-US-OMS - Cartella 73 - Messaggio cifrato in arrivo da Salamanca del 30 aprile 1937.
} 
PER VELARDI COMANDO AVIAZIONE ALT N.5788 (.) DA MINISTERO ESTERI PERVIENE RADIO CHE MI INDUCE RITENERE CHE V.S. IN RELAZIONE MIO FOGLIO N.547O ABBIA RAPPRESENTATO DIRETTAMENTE A ROMA OBIEZIONI ET CONSIDERAZIONI PERSONALI ALT PREGO TELEGRAFARMI IN PROPOSITO ET IN CASO AFFERMATIVO DARMI COMUIICAZIONE INTEGRALE DI QUANTO TRASMESSO ET RACIONI PER LF QUALI S.V. CHE EST COMPLETAMENTE MIA DIPENDENZA AVRERBE SALTATO MIO TRAMITE ALT DORIA

Il comunicato del Comandante della CTV indirizzato al Comandante dell'Aviazione Legionaria per aver rappresentato a Roma «obiezioni et considerazioni personali». Tali "obiezioni e considerazioni" erano contenute in un promemoria per il Sottosegretario e Capo di Stato Maggiore della Regia Aeronautica il Gen. Valle. Come si può osservare nel testo, il promemoria giunse anche al Ministro degli Esteri ${ }^{42}$.

Al Generale Velardi non restò che richiedere ancora una volta al Generale Valle quale fosse « la linea di condotta gradita alla E.V. », poiché, per ordine di quest'ultimo e contrariamente a quanto ordinatogli da Roatta, inviava al Gabinetto dell'Aeronautica relazioni giornaliere.

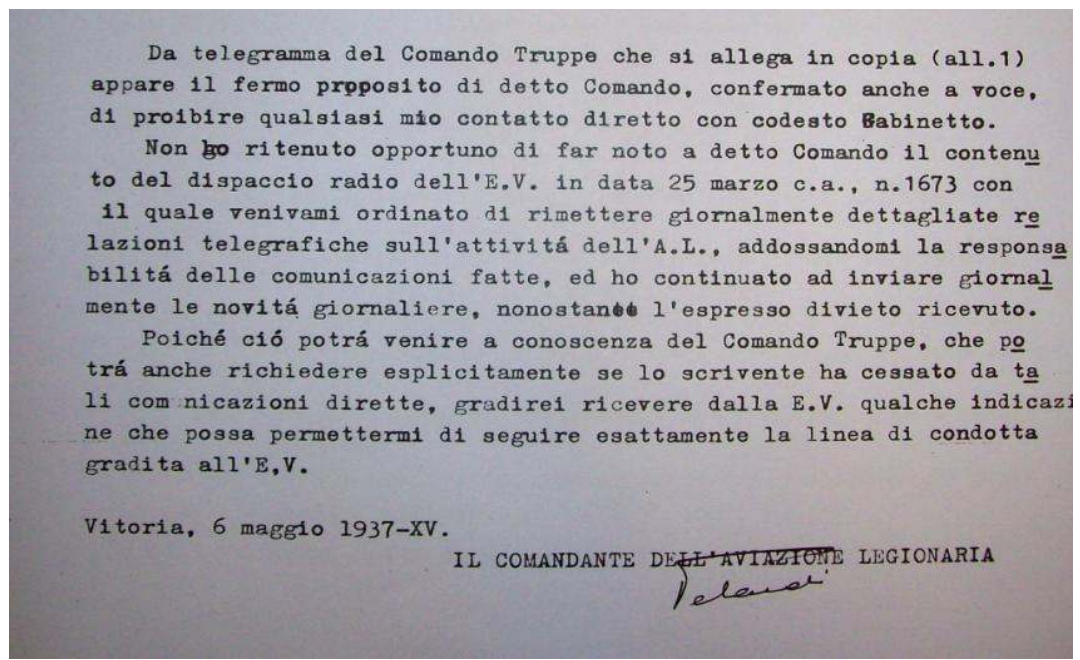

Le comunicazioni tra l'Aviazione Legionaria e il vertice della Regia Aeronautica furono formalmente proibite. Tra il Comando Aviazione Legionaria e il Gen. Valle vi fu comunque un carteggio non reso noto al Comandante del CTV. ${ }^{43}$

\footnotetext{
${ }^{42}$ SMA-US-OMS - Cartella 73.

${ }^{43}$ Ibidem.
} 
Nella breve illustrazione di questo carteggio possiamo cogliere tutta la drammaticità di una posizione di comando svolto in terra straniera e in condizioni di guerra che si trovò da una parte un Capo della Forza Armata che non procedeva a nessuna tutela delle proprie forze combattenti e, dall'altra, una linea di impiego probabilmente poco competente per cultura, tradizione e retaggio. Sorprendente, a tal proposito, anche il foglio prot. 2639 del 26 settembre 1937 a firma del Comandante CTV Generale di Corpo d'Armata Ettore Bastico con il quale si dispose che il Comando Aviazione Legionaria, in relazione al settore Zuera - Lecifiena attaccato dall'“aviazione rossa", prendesse accordi diretti con la Divisione "Frecce”, «D'ora in poi, alla botta deve darsi una risposta immediata e tale da far capire al nemico che per un dente perderà una mascella» ${ }^{44}$. Ancora una volta il comando di vertice dell'Aviazione Legionaria, doveva interloquire direttamente con comandi territoriali, Divisione "Frecce", senza un coordinamento di vertice. Lo stesso ordine di "una mascella per ogni dente" appare un giusto indicatore del livello della dottrina di impiego delle forze aeree posseduto da un Comandante del CTV.

Cercando di comprendere meglio la gerarchia del vertice militare italiano presente nel teatro operativo del conflitto civile spagnolo, con particolare riferimento all'Aviazione Legionaria, teniamo in considerazione che:

- il comando di tutte le forze terrestri e aeree, fu dato al Generale Mario Roatta, comandante della Missione Militare Italiana Spagna (MMIS) che il 16 febbraio 1937, assunse il nome di Corpo Truppe Volontario (CTV). Al comando del CTV dopo Mario Roatta seguirono, nell'ordine, il Generale di Corpo d'Armata Ettore Bastico, dall'aprile 1937, e il Generale Mario Berti, dal dicembre 1937. Nell'autunno 1938, con i primi massicci rientri delle forze italiane, il CTV venne sciolto. I compiti e le prerogative del suo Comandante furono assunti da Gastone Gambara, appena promosso al grado di Generale. Per lui, non esistendo più il CTV, fu istituita la figura del "Capo di Stato Maggiore del Generalissimo Franco per le truppe legionarie e miste";

- alle dipendenze di questi, in qualità di comandanti dell'Aviazione Legionaria, vi furono il Col. Bonomi, il Generale Bernasconi e il Generale Velardi. Tutti e quattro i Generali che si avvicendarono al Comando del CTV furono indicati da Mussolini, senza parere alcuno del re o del Capo di Stato Maggiore Generale, o di alte cariche militari. Per l'attribuzione dei comandi valeva l'assegnazione ad personam imposta direttamente da Mussolini.

\footnotetext{
${ }^{44}$ SMA-US-OMS - Cartella 47 - Sottocartella "B". Foglio prot. 2639 del 26 settembre 1937 concernente le "azioni aeree di ritorsione".
} 
Per quanto attiene più strettamente l'Aviazione Legionaria, oltre a vigere anche in questo caso il principio ad personam (il più volte citato caso del Ten Col. Cupini Ranieri ne costituisce esempio) fu vissuta una situazione di totale subordinazione ad un Generale del Regio Esercito che, per lo più, impiegò la potenza bellica aerea quale sostituta dell'artiglieria o dei carri armati, privandola totalmente di quella funzione strategica che gli era ormai riconosciuta al livello mondiale e, soprattutto, facendo si che il conflitto spagnolo perdesse ogni efficacia in termini di prove tecniche dei materiali e di valido addestramento per i piloti.

\section{Spagna cattiva maestra}

Alla luce di tutto quanto illustrato possiamo affermare che la dipendenza gerarchica dell'Aviazione Legionaria operante in Spagna durante il conflitto civile combattuto dal 1936 al 1939 fu scarsamente lineare e poco legata alla funzionalità delle rispettive cariche:

- $\quad$ Re (Comandante delle Forze Armate);

- Generale Badoglio (Capo di Stato Maggiore Generale);

- Generale Valle (Capo di Stato Maggiore della Regia Aeronautica e Sottosegretario di Stato).

Questi non presero parte alle decisioni dell'intervento militare e non ebbero alcun ruolo nella gestione, nel comando e nelle attività di coordinamento e controllo delle forze impiegate.

Diversamente, tanto sul piano decisionale, quanto direttamente o indirettamente sul concreto impiego della macchina bellica, si distinsero:

- Mussolini (Capo del Governo, Ministro della Guerra e della Regia Aeronautica);

- Galeazzo Ciano (Ministro degli Esteri);

- Ufficio Spagna (presso il MAE).

Questi tre organismi costituirono il vertice gerarchico sia da un punto di vista politico che militare. Oltre a nominare gli uomini di vertice con comunicazioni ad personam, non tenendo talvolta neanche conto della struttura ordinativa della sequenza dei gradi propria delle Forze Armate (come il caso del Generale di Brigata Roatta sopraordinato al Generale di Divisione Bernasconi) impartirono spesso, direttamente da Roma, ordini di operazione ai comandanti territoriali. L'Aviazione Legionaria delle Baleari fu, in sostanza, diretta personalmente da queste autorità politiche il che significava rivolgersi direttamente ai Comandanti di Stormo o di Gruppo e saltare il Comandante del CTV e quello dell'Aviazione Legionaria. 
L'Ufficio Spagna, e non un ufficio del Ministero della Guerra, «al quale dovranno essere esclusivamente indirizzate tutte le comunicazioni dal fronte spagnolo» ${ }^{45}$, secondo le disposizioni impartite da Mussolini, fu diretto da un dirigente del MAE diplomatico di carriera, il conte Luca Pietromarchi, che ebbe alle dipendenze tre Tenenti Colonnelli, uno per ciascuna Forza Armata che, però, non disponevano di particolari possibilità decisionali.

Il vertice militare in teatro operativo fu ubicato a Vitoria, nel nord-est della penisola iberica ed assegnato, come visto, a Ufficiali dell'Esercito portatori, quindi, di una cultura poco propensa a considerare le forze aeree come autonome e poco preparata per effettuarne un impiego strategico, ovvero con capacità risolutive. L'effettivo impiego dei velivoli si riassume con la lamentela, più volte esternata dai vertici dell'Aviazione Legionaria, che vedeva la forza aerea come una «sostituta a volta a volta delle artiglierie, dei carri armati o dei cammelli dell'Intendenza» ${ }^{46}$, non tenendo in considerazione ciò che l'ingegnere Giulio Douhet, già anni prima, aveva insegnato nella sua dottrina aerea che veniva discussa ed impiegata in tutto il mondo ${ }^{47}$.

Nel complesso, quindi, la campagna militare in Spagna, tanto ad un livello politicostrategico quanto a quello tecnico-militare, fu improntata su di un'organizzazione gerarchica poco chiara e poco lineare, spesso basata su decisioni estemporanee e prive di qualsiasi pianificazione ${ }^{48}$. Nonostante ciò, le forze nazionaliste assistite da quelle italiane, ottennero importanti successi militari a cui parteciparono - favorendo l'immagine dell'Italia in termini di "potenza militare" - anche le forze aeree. Proprio in questo periodo Mussolini esternava la sua soddisfazione per il fatto che gli «italiani [fossero] capaci di destare orrore per la loro aggressività anziché compiacimento come mandolinisti» ${ }^{49}$. In realtà, possiamo oggi indicare l'intervento, per quanto concerne le linee di comando e non solo, come un "cattivo insegnamento" per l'immediato futuro che considerava sempre più inevitabile un secondo conflitto mondiale. In particolare:

- il successo confermò sia a Mussolini che al Generale Valle di possedere una delle più efficenti aviazioni al mondo ${ }^{50}$; rispetto a ciò oggi sappiamo che in realtà solo l'Italia

\footnotetext{
${ }^{45}$ SMA-US-OMS - Cartella 46.

${ }^{46}$ SMA-US-OMS - Cartella 96. Relazione del Generale Squadra Aerea Francesco Pricolo.

${ }^{47}$ Tra i molti testi pubblicati, ricordiamo: DOUHET, Giulio, Il dominio dell'aria. Saggio sull'arte della guerra aerea, Roma, [s.e.], 1921.

${ }^{48}$ «La politica italiana in Spagna fu riserva personale di Mussolini e se le decisioni quotidiane erano affidate a Ciano e ai suoi subordinati, tutte quelle più importanti erano affidate al duce; molte erano prese sotto lo stimolo del momento [...].» in COVERDALE, John F., op. cit., p. 367.

${ }^{49}$ CIANO, Galeazzo, op.cit., p. 115 .

${ }^{50}$ Mussolini, in un discorso al Senato il 30 marzo 1938, affermò: «Oggi l'Aviazione italiana è una delle prime del mondo. Accanto alle aliquote ausiliarie dell'Esercito e della Marina e a quelle coloniali, è sorta finalmente l'Armata dell'Aria. Alcune migliaia di apparecchi quasi tutti
} 
dispiegò tutte le possibilità belliche, mentre le altre nazioni, Francia, Germania, Russia e Gran Bretagna, sperimentarono i propri mezzi e le proprie tecniche senza "investire" nel conflitto, nonostante avessero capacità e potenzialità belliche ed industriali di gran lunga superiori a quelle italiane $\mathrm{e}^{51}$;

- il sistema organizzativo ad personam trovò successive applicazioni. Non fu mai realizzato uno Stato Maggiore che coordinasse l'attività militare interforze nel quale potessero convogliare le culture, i retaggi e le competenze dell'Esercito, della Marina e dell'Aeronautica, nonostante Balbo, in uno studio del 1933, palesò tale necessità ${ }^{52}$;

- il conflitto spagnolo fu pensato come un importante laboratorio di dottrina e tecnica militare. Ne approfittò soprattutto la macchinaria bellica di Hitler che considerò apertamente la Spagna un “campo di manovra” per l'Europa. Lo stesso Göring, capo dell'aviazione tedesca, riferì nel corso del processo di Norimberga, che egli raccomandò a Hitler l'appoggio a Franco innanzitutto per impedire l'espansione del comunismo, e in secondo luogo per "provare la mia giovane Luftwaffe» ${ }^{53}$. Anche l'Italia effettuò minime sperimentazioni ma la sua partecipazione al conflitto non ebbe affatto questa finalità come prioritaria. Il Generale Pinna, in una delle molte relazioni rimaste inascoltate, scrisse che l'occasione spagnola doveva essere sfruttata in modo tale da far avvicendare il personale dopo sei-otto mesi in modo da permettere al maggior numero possibile l'utilissima esperienza di guerra ${ }^{54}$. Ma mentre il conflitto spagnolo si protraeva più di quanto era stato inizialmente preventivato e, soprattutto dopo la necessità da parte del regime fascista di "vendicare" la sconfitta subita a Guadalajara, l'Italia, inviò in Spagna quanti uomini e mezzi fossero necessari, senza però procedere a ripristinare le proprie scorte belliche. L’Aviazione Legionaria, con le molteplici dipendenze e le molteplici difficoltà operative dovute proprio alla sua difficile e promiscua dipendenza gerarchica, non poté, in definitiva, sviluppare le sperimentazioni che si era prefissata. L'occasione per realizzare futuri miglioramenti non fu quindi affatto sfruttata né per l'addestramento del personale, né per testare materiali e tecniche belliche.

recentissimi formano il complesso delle nostre forze aeree. (omissis)... I nostri C.R. 32, per quanto meno veloci, hanno nei cieli iberici fatto strage dei più veloci Curtiss e Rata». Disponibile al sito

URL: < http://www.dittatori.it/discorsimussolini.htm > [consultato il 20/.

${ }^{51}$ GRASSIA, Edoardo, L'Aviazione legionaria da bombardamento, op. cit., pp. 34-44.

52 PELLICCIA, Antonio, La Regia Aeronautica dalle origini alla Seconda Guerra Mondiale (1923-1943), op. cit., p. 33.

${ }^{53}$ THOMAS, Hugh, Storia della guerra civile spagnola, op. cit. Rilevanti accenni si rinvengono nelle carte dello SMA-US-OMS Cartella 96. In un documento classificato "SEGRETO" un Ufficiale Osservatore francese riferisce che tra gli aerei tedeschi c'è un gruppo sperimentale di cui si servono per provare le qualità belliche.

${ }^{54}$ Relazione del Generale S.A. Pinna, SMA-US-OMS - Cartella 97, Sottocartella A. Relazione sulla missione effettuata in OMS dal 12 al 22 maggio 1937. 
Mentre in Italia l'esperienza spagnola veniva osannata e considerata un monito per il futuro, negli altri Paesi europei vi fu una più lucida cautela interpretativa. E' il caso, per esempio, del Ten. Col. tedesco Herhudt Von Rhoden che, in maniera più realistica affermava che la campagna di Spagna costituiva, dal punto di vista bellico, uno scenario a se stante non immaginabile in un conflitto europeo ${ }^{55}$. Per l'Italia, oltre a conseguenze dovute agli errati insegnamenti teorici sopra indicati, si ebbero anche delle gravi conseguenze materiali ben illustrate del resoconto di una riunione che si tenne a Roma, presso Palazzo Venezia, giovedì 28 gennaio 1943, e relativa allo stato delle Forze Armate. Dall'inizio della Seconda Guerra Mondiale, per la prima volta, Mussolini tenne un vertice militare al quale parteciparono il Maresciallo d'Italia Ugo Cavallaro, il Capo di Stato Maggiore Generale Antonio Scuero, i Capi di Stato Maggiore delle singole Forze Armate Vittorio Ambrosio, Arturo Riccardi e Rino Corso Fougier e altri Ufficiali Generali. L'ordine del giorno: «Potenziamento delle FF.AA nell'attuale fase della guerra». In tale occasione Mussolini affermò che «[...] ora, dopo trentadue mesi di guerra, constatiamo che la situazione dell'aviazione non è soddisfacente. [...] solo 748 apparecchi sono di pronto impiego. Le cifre non bastano. C'è il problema qualitativo e questo vale non solo per gli uomini, che sono veramente magnifici, ma anche per le macchine. [...] I bombardieri sono 1351: cifra rispettabile che però si riduce a pochi tipi moderni. Gli altri sono superati. L'S.79 è buono ma vecchio. L'S.84 è sbagliato. Il Br.2O è superato» ${ }^{56}$.

La cifra dei bombardieri, 1351, fu ritenuta "rispettabile" (consideriamo che oltre 700 aerei, più alcuni motori e molto materiale di ricambio fu impiegato in Spagna e non fece mai ritorno, e che tra campagna spagnola e guerra d'Etiopia la Regia Aeronautica impiegò più di 1600 aerei); nonostante ciò pochi aerei furono ritenuti "moderni". I velivoli S.79 "Sparviero" e Br.20 "Cicogna", usati proprio in Spagna, furono giudicati vecchi e superati. L'S.84, che doveva essere proprio una evoluzione dell'S.79, fu indicato dal duce come 'sbagliato'. Forse una maggiore sperimentazione durante la guerra civile spagnola avrebbe permesso maggiori possibilità di successo della linea di velivoli della SIAI. Un ulteriore elemento da sottolineare nell'occasione della riunione di Palazzo Venezia fu la sentita necessità, da parte di Mussolini e in un momento di difficoltà, di riunire un vertice militare attorno ad un tavolo per discutere e non agire ad personam. A ben vedere, una buona dose di responsabilità dello stato della Regia

\footnotetext{
${ }^{55}$ VON RHODEN, Herhudt, «Guerra aerea o aerocooperazione?», in Rivista Aeronautica, agosto 1938, pp. 345-366.

${ }^{56}$ DE SIMONE, Cesare, Venti Angeli sopra Roma. I bombardamenti aerei sulla città eterna 19 luglio e 13 agosto 1943, Milano, Mursia, 1993, p. 37.
} 
«Aviazione Legionaria»: il comando strategico-politico e tecnico-militare delle forzee aeree italiane impiegate nel conflitto civile spagnolo

Aeronautica durante la Seconda Guerra Mondiale descritto da Mussolini il 28 gennaio 1943 è imputabile all'impegno che l'Italia assunse nel conflitto civile spagnolo. 


\section{Bibliografia}

BROWN, Harry, La guerra civile spagnola, Bologna, Il Mulino, 2000.

CIANO, Galeazzo, Diario 1937-1943, Milano, Rizzoli, 1980.

COVERDALE, John F., I fascisti italiani nella guerra civile di Spagna, RomaBari, Laterza, 1977.

DE SIMONE, Cesare, Venti Angeli sopra Roma. I bombardamenti aerei sulla città eterna 19 luglio e 13 agosto 1943, Milano, Mursia, 1993.

GRASSIA, Edoardo, L'Aviazione Legionaria da bombardamento. Spagna 19361939. Iniziare da stanotte azione violenta su Barcellona, Roma, IBN Editore, 2009.

KENNEDY, Paul, Ascesa e declino delle grandi potenze, Milano, Garzanti, 1989.

MONTANELLI, Indro, CERVI, Mario, Storia d'Italia, vol. XLII. La guerra di Spagna e il patto di Monaco, Milano, Rizzoli, 1984.

MUNZI, Ulderico, Il Generale, Costabissara, Angelo Calla Editore, 2009.

PELLICCIA, Antonio, La Regia Aeronautica dalle origini alla Seconda Guerra Mondiale (1923-1943), Roma, Stato Maggiore Aeronautica - Ufficio Storico, 1992.

RANZATO, Gabriele, L'eclissi della democrazia. La guerra civile spagnola e le sue origini 1931-1939, Torino, Bollati Boringhieri, 2004.

ROCHAT, Giorgio, Le guerre italiane 1935-1943, Torino, Einaudi, 2005.

RHODEN, Von Herhudt, «Guerra aerea o aerocooperazione?», Rivista Aeronautica, agosto 1938.

THOMAS, Hugh, Storia della guerra civile spagnola, Torino, Giulio Einaudi Editore, 1963.

\section{Fonti d'archivio}

Stato Maggiore Aeronautica $-5^{\circ}$ Reparto - Ufficio Storico

Fondo “Operazione Militare Spagna - OMS” (SMA-US-OMS). 


\section{* L'autore}

Edoardo Grassia, laureato in Sociologia (V.O.) presso l'Università "La Sapienza" di Roma, è ora studente del corso di laurea in Storia presso la stessa università. Si è occupato dell'Aviazione Legionaria da Bombardamento, da cui è nata la pubblicazione: L'Aviazione Legionaria da bombardamento (Spagna 1936-1939). Iniziare da stanotte azione violenta su Barcellona, Roma, IBN Editore, 2009. Svolge regolarmente attività di studio e di ricerca in ambito storico e storico-militare.

URL: < http://studistorici.com/progett/autori/\#Grassia >

\section{Per citare questo articolo:}

GRASSIA, Edoardo, «Barcellona, 17 e 18 marzo 1938», Diacronie. Studi di Storia Contemporanea: Spagna Anno Zero: la guerra come soluzione, 29/07/2011,

URL:< http://www.studistorici.com/2011/07/29/grassia2_numero_7/>

Diacronie Studi di Storia Contemporanea $\beta$ www.diacronie.it

Risorsa digitale indipendente a carattere storiografico. Uscita trimestrale. redazione.diacronie@hotmail.it

Comitato di redazione: Marco Abram - Giampaolo Amodei - Jacopo Bassi - Luca Bufarale - Alessandro Cattunar - Alice De Rensis Barbara Galimberti - Deborah Paci - Fausto Pietrancosta - Martina Sanna - Matteo Tomasoni - Luca Zuccolo 